\title{
SOME NEW INEQUALITIES ON GENERALIZATION OF HERMITE-HADAMARD AND BULLEN TYPE INEQUALITIES, APPLICATIONS TO TRAPEZOIDAL AND MIDPOINT FORMULA
}

\author{
IMDAT İSCAN ${ }^{1}$, TEKIN TOPLU ${ }^{2}$, AND FATİH YETGIN $^{3}$
}

\begin{abstract}
In this paper, we give a new general identity for differentiable functions. A consequence of the identity is that we obtain some new general inequalities containing all of the Hermite-Hadamard and Bullen type for functions whose derivatives in absolute value at certain power are convex. Some applications to special means of real numbers are also given. Finally, some error estimates for the trapezoidal and midpoint formula are addressed.
\end{abstract}

\section{INTRODUCTION}

Let $f: I \subseteq \mathbb{R} \rightarrow \mathbb{R}$ be a convex mapping defined on the interval $I$ of real numbers and $a, b \in I$ with $a<b$. The following double inequality:

$$
f\left(\frac{a+b}{2}\right) \leq \frac{1}{b-a} \int_{a}^{b} f(x) d x \leq \frac{f(a)+f(b)}{2}
$$

is known in the literature as the Hadamard inequality for convex mapping. Inequality (1.1) holds in the reversed direction if $f$ is concave. More information on these inequalities can be found in several papers and monographs (see $[2,3]$ ).

Definition 1.1. A function $f: I \subseteq \mathbb{R}=(-\infty,+\infty) \rightarrow \mathbb{R}$ is said to be convex if

$$
f(\lambda x+(1-\lambda) y) \leq \lambda f(x)+(1-\lambda) f(y)
$$

holds for all $x, y \in I$ and $\lambda \in[0,1]$.

Key words and phrases. Convex functions, Hermite-Hadamard type inequality, Bullen type inequality, general integral ineqaulities, trapezoidal and Midpoint formula.

2010 Mathematics Subject Classification. Primary: 26A51. Secondary: 26D07, 26D10, 26 D15.

DOI 10.46793/KgJMat2104.647I

Received: November 02, 2018.

Accepted: April 09, 2019. 
Theorem 1.1. Suppose that $f:[a, b] \rightarrow \mathbb{R}$ is a convex function on $[a, b]$. Then we have the inequalities:

$$
\begin{aligned}
f\left(\frac{a+b}{2}\right) & \leq \frac{1}{2}\left[f\left(\frac{3 a+b}{4}\right)+f\left(\frac{a+3 b}{4}\right)\right] \\
& \leq \frac{1}{b-a} \int_{a}^{b} f(x) d x \\
& \left.\leq \frac{1}{2}\left[f\left(\frac{a+b}{2}\right)+\frac{f(a)+f(b)}{2}\right)\right] \leq \frac{f(a)+f(b)}{2} .
\end{aligned}
$$

The third inequality in (1.2) is known in the literature as Bullen's inequality.

Lemma $1.1([1])$. Let $f: I^{\circ} \subset \mathbb{R} \rightarrow \mathbb{R}$ be a differentiable function on $I^{\circ}$, where $a, b \in I^{\circ}$, with $a<b$. If $f^{\prime} \in L[a, b]$, then

$$
\frac{f(a)+f(b)}{2}-\frac{1}{b-a} \int_{a}^{b} f(x) d x=\frac{b-a}{2} \int_{0}^{1}(1-2 t) f^{\prime}(t a+(1-t) b) d t .
$$

In [1] Dragomir and Agarwal established inequalities for differentiable convex functions which are related to Hadamard's inequlity as follows.

Theorem 1.2. ([1, Theorem 2.2]). Let $f: I^{\circ} \subseteq \mathbb{R} \rightarrow \mathbb{R}$ be a differentiable mapping on $I^{\circ}, a, b \in I^{\circ}$, with $a<b$. If $\left|f^{\prime}\right|$ is convex on $[a, b]$, then the following inequality holds

$$
\left|\frac{f(a)+f(b)}{2}-\frac{1}{b-a} \int_{a}^{b} f(x) d x\right| \leq \frac{b-a\left(\left|f^{\prime}(a)\right|+\left|f^{\prime}(b)\right|\right)}{8} .
$$

Theorem 1.3. ([1, Theorem 2.3]). Let $f: I^{\circ} \subseteq \mathbb{R} \rightarrow \mathbb{R}$ be a differentiable mapping on $I^{\circ}, a, b \in I^{\circ}$, with $a<b$ and let $p>1$. If the new mapping $\left|f^{\prime}\right|^{p / p-1}$ is convex on $[a, b]$, then the following inequality holds

$$
\left|\frac{f(a)+f(b)}{2}-\frac{1}{b-a} \int_{a}^{b} f(x) d x\right| \leq \frac{b-a}{2(p+1)^{\frac{1}{p}}}\left[\frac{\left|f^{\prime}(a)\right|^{p / p-1}+\left|f^{\prime}(b)\right|^{p / p-1}}{2}\right]^{(p-1) / p} .
$$

In [5], the above inequalities were generalized.

Theorem 1.4. ([5, Theorem 1 and 2]). Let $f: I \subset \mathbb{R} \rightarrow \mathbb{R}$ be a differentiable function on $I^{\circ}, a, b \in I$, with $a<b$ and $q \geq 1$. If $\left|f^{\prime}\right|^{q}$ is convex on $[a, b]$, then the following inequalities hold

$$
\left|\frac{f(a)+f(b)}{2}-\frac{1}{b-a} \int_{a}^{b} f(x) d x\right| \leq \frac{b-a}{4}\left(\frac{\left|f^{\prime}(a)\right|^{q}+\left|f^{\prime}(b)\right|^{q}}{2}\right)^{\frac{1}{q}}
$$

and

$$
\left|f\left(\frac{a+b}{2}\right)-\frac{1}{b-a} \int_{a}^{b} f(x) d x\right| \leq \frac{b-a}{4}\left(\frac{\left|f^{\prime}(a)\right|^{q}+\left|f^{\prime}(b)\right|^{q}}{2}\right)^{\frac{1}{q}} .
$$


In [4], the above inequalities were further generalized.

Theorem 1.5. ([4, Theorem 2.3 and 2.4]). Let $f: I^{\circ} \subset \mathbb{R} \rightarrow \mathbb{R}$ be a differentiable function on $I^{\circ}, a, b \in I^{\circ}$, with $a<b$ and $p>1$. If $\left|f^{\prime}\right|^{\frac{p}{p-1}}$ is convex on $[a, b]$, then the following inequalities hold

$$
\begin{aligned}
\left|\frac{1}{b-a} \int_{a}^{b} f(x) d x-f\left(\frac{a+b}{2}\right)\right| \leq & \frac{b-a}{16}\left(\frac{4}{p+1}\right)^{\frac{1}{p}} \\
& \times\left\{\left[\left|f^{\prime}(a)\right|^{p / p-1}+3\left|f^{\prime}(b)\right|^{p / p-1}\right]^{(p-1) / p}\right. \\
& \left.+\left[3\left|f^{\prime}(a)\right|^{p / p-1}+\left|f^{\prime}(b)\right|^{p / p-1}\right]^{(p-1) / p}\right\}
\end{aligned}
$$

and

$$
\left|\frac{1}{b-a} \int_{a}^{b} f(x) d x-f\left(\frac{a+b}{2}\right)\right| \leq \frac{b-a}{4}\left(\frac{4}{p+1}\right)^{\frac{1}{p}}\left(\left|f^{\prime}(a)\right|+\left|f^{\prime}(b)\right|\right) .
$$

Lemma $1.2([7])$. Let $f: I \subset \mathbb{R} \rightarrow \mathbb{R}$ be a differentiable function on $I^{\circ}$, where $a, b \in I$, with $a<b$. If $f^{\prime} \in L[a, b]$, then

$$
\begin{aligned}
& \frac{1}{2}\left[\frac{f(a)+f(b)}{2}+f\left(\frac{a+b}{2}\right)\right]-\frac{1}{b-a} \int_{a}^{b} f(x) d x \\
= & \frac{b-a}{4} \int_{0}^{1}\left(\frac{1}{2}-t\right)\left[f^{\prime}\left(t a+(1-t) \frac{a+b}{2}\right)+f^{\prime}\left(t \frac{a+b}{2}+(1-t) b\right)\right] d t .
\end{aligned}
$$

Corollary 1.1. ([7, Corollary 3.4]). Let $f: I \subset \mathbb{R} \rightarrow \mathbb{R}$ be a differentiable function on $I^{\circ}$, where $a, b \in I$, with $a<b$, and $f^{\prime} \in L[a, b]$. If $\left|f^{\prime}\right|$ is convex on $[a, b]$, then

$$
\left|\frac{1}{2}\left[\frac{f(a)+f(b)}{2}+f\left(\frac{a+b}{2}\right)\right]-\frac{1}{b-a} \int_{a}^{b} f(x) d x\right| \leq \frac{b-a}{16}\left(\left|f^{\prime}(a)\right|+\left|f^{\prime}(b)\right|\right) .
$$

Corollary 1.2. ([6, Corollary 3.3]). Let $f: I \subset \mathbb{R} \rightarrow \mathbb{R}$ be a differentiable function on $I^{\circ}, a, b \in I$, with $a<b$ and $f^{\prime} \in L[a, b]$. If $\left|f^{\prime}\right|^{q}$ is convex on $[a, b]$ for $q \geq 1$, then

$$
\begin{aligned}
& \left|\frac{1}{2}\left[\frac{f(a)+f(b)}{2}+f\left(\frac{a+b}{2}\right)\right]-\frac{1}{b-a} \int_{a}^{b} f(t) d t\right| \\
\leq & \frac{b-a}{16}\left(\frac{1}{12}\right)^{\frac{1}{q}}\left[\left(9\left|f^{\prime}(a)\right|^{q}+3\left|f^{\prime}(b)\right|^{q}\right)^{\frac{1}{q}}+\left(3\left|f^{\prime}(a)\right|^{q}+9\left|f^{\prime}(b)\right|^{q}\right)^{\frac{1}{q}}\right] .
\end{aligned}
$$

\section{Main Results}

In order to establish our main results, we first establish the following lemma. 
Lemma 2.1. Let $f: I^{\circ} \subset \mathbb{R} \rightarrow \mathbb{R}$ be a differentiable function on $I^{\circ}$, where $a, b \in I^{\circ}$, with $a<b$. If $f^{\prime} \in L[a, b]$, then the following equality holds

$$
\begin{aligned}
I_{n}(f, a, b)= & \sum_{i=0}^{n-1} \frac{1}{2 n}\left[f\left(\frac{(n-i) a+i b}{n}\right)+f\left(\frac{(n-i-1) a+(i+1) b}{n}\right)\right] \\
& -\frac{1}{b-a} \int_{a}^{b} f(x) d x \\
= & \sum_{i=0}^{n-1} \frac{b-a}{2 n^{2}}\left[\int _ { 0 } ^ { 1 } ( 1 - 2 t ) f ^ { \prime } \left(t \frac{(n-i) a+i b}{n}\right.\right. \\
& \left.\left.+(1-t) \frac{(n-i-1) a+(i+1) b}{n}\right) d t\right] .
\end{aligned}
$$

Proof. If we take $n \in \mathbb{N}$ arbitrarily, then for $i \in\{1,2, \ldots, n-1\}$ by integration by parts, we have

$$
\begin{aligned}
I_{i}= & \int_{0}^{1}(1-2 t) f^{\prime}\left(t \frac{(n-i) a+i b}{n}+(1-t) \frac{(n-i-1) a+(i+1) b}{n}\right) d t \\
= & \left.\frac{n}{a-b}(1-2 t) f\left(t \frac{(n-i) a+i b}{n}+(1-t) \frac{(n-i-1) a+(i+1) b}{n}\right)\right|_{0} ^{1} \\
& +\frac{2 n}{a-b} \int_{0}^{1} f\left(t \frac{(n-i) a+i b}{n}+(1-t) \frac{(n-i-1) a+(i+1) b}{n}\right) d t
\end{aligned}
$$

By making use of the substitutions $x=t \frac{(n-i) a+i b}{n}+(1-t) \frac{(n-i-1) a+(i+1) b}{n}$

$$
\begin{aligned}
I_{i}= & -\frac{n}{a-b}\left[f\left(\frac{(n-i) a+i b}{n}\right)+f\left(\frac{(n-i-1) a+(i+1) b}{n}\right)\right] \\
& -\frac{2 n^{2}}{(a-b)^{2}} \int_{\frac{(n-i) a+i b}{n}}^{\frac{(n-i-1) a+(i+1) b}{n}} f(x) d x .
\end{aligned}
$$

Multiplying the both sides by $\frac{b-a}{2 n^{2}}$, we have

$$
\begin{aligned}
\frac{b-a}{2 n^{2}} I_{i}= & \frac{1}{2 n}\left[f\left(\frac{(n-i) a+i b}{n}\right)+f\left(\frac{(n-i-1) a+(i+1) b}{n}\right)\right] \\
& -\frac{1}{b-a} \int_{\frac{(n-i) a+i b}{n}}^{\frac{(n-i-1) a+(i+1) b}{n}} f(x) d x .
\end{aligned}
$$


Finally, we have

$$
\begin{aligned}
\sum_{i=0}^{n-1} \frac{b-a}{2 n^{2}} I_{i}= & \sum_{i=0}^{n-1} \frac{1}{2 n}\left[f\left(\frac{(n-i) a+i b}{n}\right)+f\left(\frac{(n-i-1) a+(i+1) b}{n}\right)\right] \\
& -\frac{1}{b-a} \sum_{i=0}^{n-1} \int_{\frac{(n-i) a+i b}{n}}^{\frac{(n-i) a+(i+1) b}{n}} f(x) d x \\
= & \sum_{i=0}^{n-1} \frac{1}{2 n}\left[f\left(\frac{(n-i) a+i b}{n}\right)+f\left(\frac{(n-i-1) a+(i+1) b}{n}\right)\right] \\
& -\frac{1}{b-a} \int_{a}^{b} f(x) d x
\end{aligned}
$$

Remark 2.1. If we choose $\mathrm{n}=1$ in Lemma 2.1, then (2.1) reduces to (1.3).

Remark 2.2. If we choose $\mathrm{n}=2$ in Lemma 2.1, then (2.1) reduces to (1.7).

Theorem 2.1. Let $f: I \subset \mathbb{R} \rightarrow \mathbb{R}$ be a differentiable function on $I^{\circ}$, where $a, b \in I^{\circ}$, with $a<b$. If $\left|f^{\prime}\right|^{q}$ is convex on $[a, b]$ for some fixed $q \geq 1$, then the following inequality is satisfied

$$
\left|I_{n}(f, a, b)\right| \leq \sum_{i=0}^{n-1} \frac{b-a}{4 n^{2}}\left[\left(\frac{2 n-2 i-1}{2 n}\right)\left|f^{\prime}(a)\right|^{q}+\left(\frac{2 i+1}{2 n}\right)\left|f^{\prime}(b)\right|^{q}\right]^{\frac{1}{q}} .
$$

Proof. From Lemma 2.1 and by using the well known Power-mean inequality, we have

$$
\begin{aligned}
& \left|I_{n}(f, a, b)\right| \\
\leq & \sum_{i=0}^{n-1} \frac{b-a}{2 n^{2}}\left(\int_{0}^{1}|1-2 t| d t\right)^{1-\frac{1}{q}} \\
& \times\left(\int_{0}^{1}|1-2 t|\left|f^{\prime}\left(t \frac{(n-i) a+i b}{n}+(1-t) \frac{(n-i-1) a+(i+1) b}{n}\right)\right|^{q} d t\right)^{\frac{1}{q}}
\end{aligned}
$$

By using the convexity of $\left|f^{\prime}\right|^{q}$, we have

$$
\begin{aligned}
& \left|I_{n}(f, a, b)\right| \\
\leq & \sum_{i=0}^{n-1} \frac{b-a}{2 n^{2}}\left[\int_{0}^{1}|1-2 t| d t\right]^{1-\frac{1}{q}}\left[\int _ { 0 } ^ { 1 } | 1 - 2 t | \left(t\left|f^{\prime}\left(\frac{(n-i) a+i b}{n}\right)\right|^{q}\right.\right. \\
& \left.\left.+(1-t) \cdot\left|f^{\prime}\left(\frac{(n-i-1) a+(i+1) b}{n}\right)\right|^{q}\right) d t\right]^{\frac{1}{q}}
\end{aligned}
$$




$$
\begin{aligned}
= & \sum_{i=0}^{n-1} \frac{b-a}{2 n^{2}}\left(\int_{0}^{1}|1-2 t| d t\right)^{1-\frac{1}{q}}\left(\int_{0}^{1}|1-2 t| t .\left|f^{\prime}\left(\frac{(n-i) a+i b}{n}\right)\right|^{q} d t\right. \\
& \left.+\int_{0}^{1}|1-2 t|(1-t)\left|f^{\prime}\left(\frac{(n-i-1) a+(i+1) b}{n}\right)\right|^{q} d t\right)^{\frac{1}{q}} \\
= & \sum_{i=0}^{n-1} \frac{b-a}{2 n^{2}}\left[\left(\frac{1}{2}\right)^{1-\frac{1}{q}}\left(\frac{\left|f^{\prime}\left(\frac{(n-i) a+i b}{n}\right)\right|^{q}+f^{\prime}\left|\left(\frac{(n-i-1) a+(i+1) b}{n}\right)\right|^{q}}{4}\right)^{\frac{1}{q}}\right] \\
= & \sum_{i=0}^{n-1} \frac{b-a}{n^{2}(2)^{2+\frac{1}{q}}}\left(\left|f^{\prime}\left(\frac{(n-i) a+i b}{n}\right)\right|^{q}+\left|f^{\prime}\left(\frac{(n-i-1) a+(i+1) b}{n}\right)\right|^{q}\right)^{\frac{1}{q}}
\end{aligned}
$$

Finally, by using the convexity of $\left|f^{\prime}\right|^{q}$, we have

$$
\left|I_{n}(f, a, b)\right| \leq \sum_{i=0}^{n-1} \frac{b-a}{n^{2}(2)^{2+\frac{1}{q}}}\left[\left(\frac{2 n-2 i-1}{n}\right)\left|f^{\prime}(a)\right|^{q}+\left(\frac{2 i+1}{n}\right)\left|f^{\prime}(b)\right|^{q}\right]^{\frac{1}{q}} .
$$

Remark 2.3. If we choose $n=1$ in Theorem 2.1, then (2.2) reduces to (1.6).

Remark 2.4. If we choose $n=1$ and $q=1$ in Theorem 2.1, then (2.2) reduces to (1.4).

Remark 2.5. If we choose $n=2$ in Theorem 2.1, then (2.2) reduces to (1.9).

Remark 2.6. If we choose $n=2$ and $q=1$ in Theorem 2.1, then (2.2) reduces to (1.8).

Corollary 2.1. If we choose $n=3$ in Theorem 2.1, then we obtain

$$
\begin{aligned}
& \left|\frac{1}{6}\left[f(a)+f(b)+2 f\left(\frac{2 a+b}{3}\right)+2 f\left(\frac{a+2 b}{3}\right)\right]-\frac{1}{b-a} \int_{0}^{1} f(x) d x\right| \\
\leq & \frac{b-a}{6^{2+\frac{1}{q}}}\left[\left(5\left|f^{\prime}(a)\right|^{q}+\left|f^{\prime}(b)\right|^{q}\right)^{\frac{1}{q}}+\left(3\left|f^{\prime}(a)\right|^{q}+3\left|f^{\prime}(b)\right|^{q}\right)^{\frac{1}{q}}+\left(\left|f^{\prime}(a)\right|^{q}+5\left|f^{\prime}(b)\right|^{q}\right)^{\frac{1}{q}}\right] .
\end{aligned}
$$

Theorem 2.2. Let $f: I \subset \mathbb{R} \rightarrow \mathbb{R}$ be a differentiable function on $I^{\circ}$, where $a, b \in I^{\circ}$, with $a<b$. If $\left|f^{\prime}\right|^{q}$ is convex on $[a, b]$ for some fixed $q>1$, then the following inequality is satisfied

$$
\left|I_{n}(f, a, b)\right| \leq \sum_{i=0}^{n-1} \frac{b-a}{n^{2} 2^{1+\frac{1}{q}}}\left(\frac{1}{p+1}\right)^{\frac{1}{p}}\left[\left(\frac{2 n-2 i-1}{n}\right)\left|f^{\prime}(a)\right|^{q}+\left(\frac{2 i+1}{n}\right)\left|f^{\prime}(b)\right|^{q}\right]^{\frac{1}{q}}
$$

where $\frac{1}{q}+\frac{1}{p}=1$.

Proof. From Lemma 2.1 and by using the Hölder inequality, we have 


$$
\begin{aligned}
\left|I_{n}(f, a, b)\right| \leq & \sum_{i=0}^{n-1} \frac{b-a}{2 n^{2}}\left[\left(\int_{0}^{1}|1-2 t|^{p} d t\right)^{\frac{1}{p}}\right. \\
& \left.\times\left(\int_{0}^{1}\left|f^{\prime}\left(t \frac{(n-i) a+i b}{n}+(1-t) \frac{(n-i-1) a+(i+1) b}{n}\right)\right|^{q} d t\right)^{\frac{1}{q}}\right]
\end{aligned}
$$

By using the convexity of $\left|f^{\prime}\right|^{q}$, we have

$$
\begin{aligned}
& \left|I_{n}(f, a, b)\right| \\
\leq & \sum_{i=0}^{n-1} \frac{b-a}{2 n^{2}}\left[\left(\int_{0}^{1}|1-2 t|^{p} d t\right)^{\frac{1}{p}}\right. \\
& \left.\times\left(\int_{0}^{1}\left(t\left|f^{\prime}\left(\frac{(n-i) a+i b}{n}\right)\right|^{q}+(1-t)\left|f^{\prime}\left(\frac{(n-i-1) a+(i+1) b}{n}\right)\right|^{q}\right) d t\right)^{\frac{1}{q}}\right] \\
= & \sum_{i=0}^{n-1} \frac{b-a}{2^{1+\frac{1}{q}} n^{2}}\left[\left(\frac{1}{p+1}\right)^{\frac{1}{p}}\right. \\
& \left.\times\left(\left|f^{\prime}\left(\frac{(n-i) a+i b}{n}\right)\right|^{q}+\left|f^{\prime}\left(\frac{(n-i-1) a+(i+1) b}{n}\right)\right|^{q}\right)^{\frac{1}{q}}\right] .
\end{aligned}
$$

By using the convexity of $\left|f^{\prime}\right|^{q}$, we have

$$
\left|I_{n}(f, a, b)\right| \leq \sum_{i=0}^{n-1} \frac{b-a}{n^{2} 2^{1+\frac{1}{q}}}\left(\frac{1}{p+1}\right)^{\frac{1}{p}}\left[\left(\frac{2 n-2 i-1}{n}\right)\left|f^{\prime}(a)\right|^{q}+\left(\frac{2 i+1}{n}\right)\left|f^{\prime}(b)\right|^{q}\right]^{\frac{1}{q}}
$$

Remark 2.7. If we choose $n=1$ in Theorem 2.2, then (2.3) reduces to (1.5).

Corollary 2.2. If we choose $n=2$ in Theorem 2.2, then we have

$$
\begin{aligned}
& \left|\frac{1}{2}\left[\frac{f(a)+f(b)}{2}+f\left(\frac{a+b}{2}\right)\right]-\frac{1}{b-a} \int_{a}^{b} f(x) d x\right| \\
= & \frac{b-a}{2^{3+\frac{2}{q}}}\left(\frac{1}{p+1}\right)^{\frac{1}{p}}\left[\left(3\left|f^{\prime}(a)\right|^{q}+\left|f^{\prime}(b)\right|\right)^{\frac{1}{q}}+\left(\left|f^{\prime}(a)\right|^{q}+3\left|f^{\prime}(b)\right|^{q}\right)^{\frac{1}{q}}\right] .
\end{aligned}
$$

Corollary 2.3. If we choose $n=3$ in Theorem 2.2, then we have

$$
\begin{aligned}
& \left|\frac{1}{6}\left[f(a)+f(b)+2 f\left(\frac{2 a+b}{3}\right)+2 f\left(\frac{a+2 b}{3}\right)\right]-\frac{1}{b-a} \int_{0}^{1} f(x) d x\right| \\
\leq & \frac{b-a}{18 \cdot 6^{\frac{1}{q}}}\left(\frac{1}{p+1}\right)^{\frac{1}{p}}\left[\left(5\left|f^{\prime}(a)\right|^{q}+\left|f^{\prime}(b)\right|^{q}\right)^{\frac{1}{q}}+\left(3\left|f^{\prime}(a)\right|^{q}+3\left|f^{\prime}(b)\right|^{q}\right)^{\frac{1}{q}}\right.
\end{aligned}
$$




$$
\left.+\left(\left|f^{\prime}(a)\right|^{q}+5\left|f^{\prime}(b)\right|^{q}\right)^{\frac{1}{q}}\right]
$$

\section{Applications to Special Means}

We consider some special means, for which will get new inequalities. Let $a, b \in \mathbb{R}$.

(i) The arithmetic mean: $A=A(a, b):=\frac{a+b}{2}, a, b \geq 0$.

(ii) The harmonic mean:

$$
H=H(a, b):=\frac{2 a b}{a+b}, \quad a, b>0 .
$$

(iii) The logarithmic mean:

$$
L=L(a, b):=\left\{\begin{array}{ll}
a, & \text { if } a=b, \\
\frac{b-a}{\ln b-\ln a}, & \text { if } a \neq b,
\end{array} \quad a, b>0 .\right.
$$

(iv) The p-logarithmic mean

$$
L_{p}=L_{p}(a, b):=\left\{\begin{array}{ll}
a, & \text { if } a=b, \\
{\left[\frac{b^{p+1}-a^{p+1}}{(p+1)(b-a)}\right]^{\frac{1}{p}},} & \text { if } a \neq b,
\end{array} \quad p \in \mathbb{R} \backslash\{-1,0\}, a, b>0 .\right.
$$

Proposition 3.1. Let $a, b \in \mathbb{R}, 0<a<b$ and $t \in \mathbb{N}, t \geq 2$. Then, for all $q \geq 1$, the following inequality holds

$$
\begin{aligned}
& \left|\sum_{i=0}^{n-1} \frac{1}{n} A\left(\left(\frac{(n-i) a+i b}{n}\right)^{t},\left(\frac{(n-i-1) a+(i+1) b}{n}\right)^{t}\right)-L_{t}^{t}(a, b)\right| \\
\leq & \sum_{i=0}^{n-1} \frac{(b-a) t}{n^{2}(2)^{2+\frac{1}{q}}}\left[\left(\frac{(2 n-2 i-1)}{n}\right) a^{(t-1) q}+\left(\frac{2 i+1}{n}\right) b^{(t-1) q}\right]^{\frac{1}{q}} .
\end{aligned}
$$

Proof. The proof is immediate from (2.2) in Theorem 2.1, with $f(x)=x^{t}, x \in[a, b]$, $t \in \mathbb{N}, t \geq 2$.

Remark 3.1. (a) If we choose $n=1$, in the Proposition 3.1, we have [5, Proposition 1] for positive real numbers.

(b) If we choose $n=1$ and $q=1$, in the Proposition 3.1, we have [1, Proposition 3.1] for positive real numbers.

Proposition 3.2. Let $a, b \in \mathbb{R}, 0<a<b$ and $t \in \mathbb{N}, t \geq 2$. Then, for all $q>1$, the following inequality holds

$$
\begin{aligned}
& \left|\sum_{i=0}^{n-1} \frac{1}{n} A\left(\left(\frac{(n-i) a+i b}{n}\right)^{t},\left(\frac{(n-i-1) a+(i+1) b}{n}\right)\right)-L_{t}^{t}(a, b)\right| \\
\leq & \sum_{i=0}^{n-1} \frac{(b-a) t}{n^{2} 2^{1+\frac{1}{q}}}\left(\frac{1}{p+1}\right)^{\frac{1}{p}}\left[\left(\frac{(2 n-2 i-1)}{n}\right) a^{(t-1) q}+\left(\frac{2 i+1}{n}\right) b^{(t-1) q}\right]^{\frac{1}{q}} .
\end{aligned}
$$


Proof. The proof is immediate from (2.3) in Theorem 2.2, with $f(x)=x^{t}, x \in[a, b]$, $t \in \mathbb{N}, t \geq 2$.

Remark 3.2. If we choose $n=1$, in the Proposition 3.2, we have [1, Proposition 3.2] for positive real numbers.

Proposition 3.3. Suppose $a, b \in \mathbb{R}, 0<a<b$. Then, for all $q \geq 1$, the following inequality holds

$$
\begin{aligned}
& \left|\sum_{i=0}^{n-1} \frac{1}{n} H^{-1}\left(\left(\frac{(n-i) a+i b}{n}\right),\left(\frac{(n-i-1) a+(i+1) b}{n}\right)\right)-L^{-1}(a, b)\right| \\
\leq & \sum_{i=0}^{n-1} \frac{(b-a)}{n^{2}(2)^{2+\frac{1}{q}}}\left[\left(\frac{(2 n-2 i-1)}{n}\right) a^{-2 q}+\left(\frac{2 i+1}{n}\right) b^{-2 q}\right]^{\frac{1}{q}} .
\end{aligned}
$$

Proof. The proof is immediate from (2.2) in Theorem 2.1, with $f(x)=\frac{1}{x}, x \in[a, b]$.

Remark 3.3. (a) If we choose $n=1$, in the Proposition 3.3, we have [5, Proposition 2] for positive real numbers.

(b) If we choose $n=1$ and $q=1$, in the Proposition 3.3, we have [1, Proposition 3.3] for positive real numbers.

Proposition 3.4. Let $a, b \in \mathbb{R}, 0<a<b$. Then, for all $q>1$, the following inequality holds

$$
\begin{aligned}
& \left|\sum_{i=0}^{n-1} \frac{1}{n} H^{-1}\left(\left(\frac{(n-i) a+i b}{n}\right),\left(\frac{(n-i-1) a+(i+1) b}{n}\right)\right)-L^{-1}(a, b)\right| \\
\leq & \sum_{i=0}^{n-1} \frac{(b-a)}{n^{2} 2^{1+\frac{1}{q}}}\left(\frac{1}{p+1}\right)^{\frac{1}{p}}\left[\left(\frac{(2 n-2 i-1)}{n}\right) a^{-2 q}+\left(\frac{2 i+1}{n}\right) b^{-2 q}\right]^{\frac{1}{q}} .
\end{aligned}
$$

Proof. The proof is immediate from (2.3) in Theorem 2.2, with $f(x)=\frac{1}{x}, x \in[a, b]$.

Remark 3.4. If we choose $n=1$, in the Proposition 3.4, we have [1, Proposition 3.4] for positive real numbers.

\section{Applications to Trapezoided and Midpoint Formulas}

Throughout this section, let $f:[a, b] \rightarrow \mathbb{R}$ be integrable and let $I_{t}: a=x_{0}<x_{1}<$ $\cdots<x_{t}=b$ be a partition of $[a, b]$ and $l_{k}=x_{k+1}-x_{k}, k=0,1, \ldots, t-1$. Tseng et al. described the following notations in [8]:

- the trapezoidal formula

$$
T\left(f, I_{t}\right)=\sum_{k=0}^{t-1} \frac{f\left(x_{k}\right)+f\left(x_{k+1}\right)}{2} l_{k}
$$

- the midpoint formula

$$
M\left(f, I_{t}\right)=\sum_{k=0}^{t-1} f\left(\frac{x_{k}+x_{k+1}}{2}\right) l_{k}
$$


- the approximation error of $\int_{a}^{b} f(x) d x$ by $T\left(f, I_{t}\right)$

$$
E\left(f, I_{t}\right)=\int_{a}^{b} f(x) d x-T\left(f, I_{t}\right)
$$

- the approximation error of $\int_{a}^{b} f(x) d x$ by $M\left(f, I_{t}\right)$

$$
F\left(f, I_{t}\right)=\int_{a}^{b} f(x) d x-M\left(f, I_{t}\right) .
$$

In [5] Pearce and Pecaric established the following proposition which is approximation errors for the trapezoidal and midpoint formulas.

Proposition 4.1. Under the conditions of Theorem 1.4, we have the following inequalities

$$
\left|E\left(f, I_{t}\right)\right| \leq \frac{1}{4} \sum_{k=0}^{t-1}\left(\frac{\left|f^{\prime}\left(x_{k}\right)\right|^{q}+\left|f^{\prime}\left(x_{k+1}\right)\right|^{q}}{2}\right)^{\frac{1}{q}} l_{k}^{2} \leq \frac{\max \left\{\left|f^{\prime}(a)\right|,\left|f^{\prime}(b)\right|\right\}}{4} \sum_{k=0}^{t-1} l_{k}^{2}
$$

and

$$
\left|F\left(f, I_{t}\right)\right| \leq \frac{1}{4} \sum_{k=0}^{t-1}\left(\frac{\left|f^{\prime}\left(x_{k}\right)\right|^{q}+\left|f^{\prime}\left(x_{k+1}\right)\right|^{q}}{2}\right)^{\frac{1}{q}} l_{k}^{2} \leq \frac{\max \left\{\left|f^{\prime}(a)\right|,\left|f^{\prime}(b)\right|\right\}}{4} \sum_{k=0}^{t-1} l_{k}^{2} .
$$

We have the following proposition which reduce (4.1) in Propositions 4.1 as $n=1$ on $[a, b]$.

Proposition 4.2. Under the conditions of Theorem 2.1, we have the following inequalities

$$
\begin{aligned}
& \mid \sum_{k=0}^{t-1} \sum_{i=0}^{n-1} \frac{1}{2 n}\left[f\left(\frac{(n-i) x_{k}+i x_{k+1}}{n}\right)+f\left(\frac{(n-i-1) x_{k}+(i+1) x_{k+1}}{n}\right)\right]\left(x_{k+1}-x_{k}\right) \\
& -\int_{a}^{b} f(x) d x \mid \leq \sum_{k=0}^{t-1} \frac{\left(x_{k+1}-x_{k}\right)^{2}}{4 n^{2}} \sum_{i=0}^{n-1}\left[\left(\frac{2 n-2 i-1}{2 n}\right)\left|f^{\prime}\left(x_{k}\right)\right|^{q}+\left(\frac{2 i+1}{2 n}\right)\left|f^{\prime}\left(x_{k+1}\right)\right|^{q}\right]^{\frac{1}{q}}
\end{aligned}
$$

$$
\leq \frac{1}{4 n} \max \left\{\left|f^{\prime}(a)\right|,\left|f^{\prime}(b)\right|\right\} \sum_{k=0}^{t-1}\left(x_{k+1}-x_{k}\right)^{2} \text {. }
$$

Proof. Apply Theorem 2.1 on $\left[x_{k}, x_{k+1}\right], k=0,1, \ldots, t-1$, we get

$$
\begin{aligned}
& \mid \sum_{i=0}^{n-1} \frac{1}{2 n}\left[f\left(\frac{(n-i) x_{k}+i x_{k+1}}{n}\right)+f\left(\frac{(n-i-1) x_{k}+(i+1) x_{k+1}}{n}\right)\right]\left(x_{k+1}-x_{k}\right) \\
& -\int_{x_{k}}^{x_{k+1}} f(x) d x \mid \leq \frac{\left(x_{k+1}-x_{k}\right)^{2}}{4 n^{2}} \sum_{i=0}^{n-1}\left[\left(\frac{2 n-2 i-1}{2 n}\right)\left|f^{\prime}\left(x_{k}\right)\right|^{q}+\left(\frac{2 i+1}{2 n}\right)\left|f^{\prime}\left(x_{k+1}\right)\right|^{q}\right]^{\frac{1}{q}} .
\end{aligned}
$$

Taking into account that $\left|f^{\prime}\right|^{q}$ is convex, we deduce, by the triangle inequality, that

$$
\left[\left(\frac{2 n-2 i-1}{2 n}\right)\left|f^{\prime}\left(x_{k}\right)\right|^{q}+\left(\frac{2 i+1}{2 n}\right)\left|f^{\prime}\left(x_{k+1}\right)\right|^{q}\right] \leq \max \left\{\left|f^{\prime}(a)\right|^{q},\left|f^{\prime}(b)\right|^{q}\right\} .
$$


Finally, summing over $k$ from 0 to $t-1$, we have (4.2).

Remark 4.1. If we choose $n=1$ on $[a, b]$, then the (4.2) reduce to (4.1).

Corollary 4.1. If we choose $n=2$ in Proposition 4.2, we get

$$
\begin{aligned}
& \left|E\left(f, I_{t}\right)+F\left(f, I_{t}\right)\right| \\
\leq & \sum_{k=0}^{t-1} \frac{\left(x_{k+1}-x_{k}\right)^{2}}{8}\left[\left(\frac{3}{4}\left|f^{\prime}\left(x_{k}\right)\right|^{q}+\frac{1}{4}\left|f^{\prime}\left(x_{k+1}\right)\right|\right)^{\frac{1}{q}}+\left(\frac{1}{4}\left|f^{\prime}\left(x_{k}\right)\right|^{q}+\frac{3}{4}\left|f^{\prime}\left(x_{k+1}\right)\right|^{q}\right)^{\frac{1}{q}}\right] \\
\leq & \frac{1}{4} \max \left\{\left|f^{\prime}(a)\right|,\left|f^{\prime}(b)\right|\right\} \sum_{k=0}^{t-1}\left(x_{k+1}-x_{k}\right)^{2} .
\end{aligned}
$$

\section{REFERENCES}

[1] S. S. Dragomir and R. P. Agarwal, Two inequalities for differentiable mappings and applications to special means of real numbers and to trapezoidal formula, Appl. Math. Lett. 11 (1998), 91-95.

[2] S. S. Dragomir and C. E. M. Pearce, Selected Topics on Hermite-Hadamard Inequalities and Applications, RGMIA Monographs, Victoria University, Melbourne, 2000.

[3] J. Pečarić, F. Proschan and Y. L. Tong, Convex Functions, Partial Ordering and Statistical Applications, Academic Press, San Diego, 1992.

[4] U. S. Kirmaci, Inequalities for differentiable mappings and applications to special means of real numbers to midpoint formula, Appl. Math. Comput. 147 (2004), 137-146.

[5] C. E. M. Pearce and J. Pečarić, Inequalities for differentiable mappings with application to special means and quadrature formulae, Appl. Math. Lett. 13 (2000), 51-55.

[6] B.-Y. Xi and F. Qi, Some integral inequalities of Hermite-Hadamard type for convex functions with applications to means, Journal of Function Spaces and Applications (2012), Article ID 980438.

[7] B.-Y. Xi and F. Qi, Some Hermite-Hadamard type inequalities for differentiable convex functions and applications, Hacet. J. Math. Stat. 42 (2013), 243-257.

[8] K-L. Tseng, S-R. Hwang, G.-S. Yang and J.-C. Lo, Two inequalities for differentiable mappings and applications to weighted trapezoidal formula, weighted midpoint formula and random variable, Math. Comput. Modelling 53 (2011), 179-188.

${ }^{1}$ Department of Mathematics Faculty of Arts and Sciences,

Giresun University,

28200, Giresun, Turkey

Email address: imdat.iscan@giresun.edu.tr

${ }^{2}$ Department of Mathematics Institute of Science,

Giresun University,

28200, Giresun, Turkey

Email address: tekintoplu@gmail.com

${ }^{3}$ Department of Mathematics Faculty of Basic Seciences,

Gebze TeChNicAl University,

41400 Gebze-KocAeli, TuRKey

Email address: fyetgin@gtu.edu.tr 